\title{
Calcul des contraintes résiduelles dans les zones d'assemblage de plaques en verre trempé
}

\author{
Fabrice Bernard ${ }^{1}$, Laurent Daudeville ${ }^{2, a}$ et René Gy $^{3}$ \\ 1 École des mines de Douai, Département Génie Civil, 941 rue Charles Bourseul, 59508 Douai Cedex, France \\ 2 Laboratoire Sols Solides Structures, Domaine Universitaire, 38041 Grenoble Cedex 9, France \\ 3 Saint-Gobain Recherche, 39 quai Lucien Lefranc, 93303 Aubervilliers Cedex, France
}

Reçu le 2 juillet 2004, accepté le 5 novembre 2004

\begin{abstract}
Résumé - Ce travail porte sur l'utilisation du verre comme véritable matériau de structure et concerne plus particulièrement le calcul des contraintes résiduelles de trempe autour des zones d'assemblage par insertion d'un connecteur métallique au sein de plaques trouées et chanfreinées pour la prédiction de la résistance de tels assemblages. Des simulations numériques par la méthode des éléments finis du procédé de trempe sont menées pour le calcul des contraintes transitoires et résiduelles près des bords de plaques de verre (calculs $2 \mathrm{D}$ ) et près de trous chanfreinés (calculs 3D). Le verre est considéré comme un matériau viscoélastique, le modèle de Narayanaswamy est utilisé, il prend en compte les phénomènes de relaxation de contraintes d'origine visqueuse et structurale. Une difficulté provient de la description correcte des transferts de chaleur pendant la trempe qui est d'importance majeure pour la prédiction des contraintes résiduelles. La convection forcée due au soufflage de l'air et le rayonnement sont modélisés, le caractère semi-transparent du verre est pris en compte. Les coefficients de convection forcée dans les zones bords sont identifiés expérimentalement. Les résultats de prédiction des contraintes résiduelles de trempe sont comparés à des résultats de mesures photo-élastiques. Ce travail permet l'optimisation de la géométrie de la plaque de verre pour un renforcement maximal dans la zone d'assemblage.
\end{abstract}

Mots clés : Verre trempé / structures / photoélasticité / éléments finis / contraintes résiduelles / assemblages

\begin{abstract}
Prediction of residual stresses near joints in tempered glass plates. This work deals with the use of glass as a real structural material and concerns more particularly the calculation of residual stresses due to tempering in the vicinity of dowel type joints inserted into chamfered holes in the aim of predicting the load bearing capacity of such joints. This work presents numerical simulation results of the thermal tempering by the Finite Element Method in order to calculate transient and residual stresses near edges of a glass plate (2D calculation) and near holes (3D calculation). During thermal tempering, glass is considered as a viscoelastic material. The Narayanaswamy's model is used. It takes into account the structural relaxation phenomena. The particular difficulty is the correct modelling of heat transfers since transient and residual stresses strongly depend on the history of temperature within the plate, close to the edge and to the hole. Both the forced convection due to the blowing of air and the radiative heat transfer are modeled numerically. The semi-transparency of glass in the near infrared range is considered. The convective heat transfer coefficients on the edge and hole walls are identified thanks to a specific experimental set-up and validated from simulations of heat transfer tests. The computed residual stresses are checked against photo-elastic measurements. Such a work allows the optimization of the glass plate geometry in the vicinity of the joint in the aim of a maximum reinforcement.
\end{abstract}

Key words: Tempered glass / structures / photoelasticity / finite elements / residual stresses / joints

a Auteur correspondant : laurent.daudeville@inpg.fr 


\section{Nomenclature}

\begin{tabular}{|c|c|}
\hline$A$ & paramètre de la loi de Vogel-Fulcher-Tamman \\
\hline$B$ & paramètre de la loi de Vogel-Fulcher-Tamman \\
\hline$c_{\mathrm{p}}$ & chaleur spécifique massique de l'aluminium \\
\hline$e$ & épaisseur de la plaque de verre \\
\hline$G$ & module de relaxation de cisaillement \\
\hline$G_{\mathrm{g}}$ & module de relaxation instantanée de cisaillement \\
\hline$h$ & coefficient de convection forcée \\
\hline$K$ & module de relaxation en pression hydrostatique \\
\hline$K_{\mathrm{e}}$ & module de relaxation différée en pression hydrostatique \\
\hline$K_{\mathrm{g}}$ & module de relaxation instantanée en pression hydrostatique \\
\hline$M_{\mathrm{v}}$ & fonction relaxation de volume \\
\hline$T$ & température \\
\hline$t$ & temps \\
\hline$T_{0}$ & température initiale de la plaque de verre \\
\hline$T_{\text {air }}$ & température de l'air soufflé \\
\hline$T_{\text {ext }}$ & température de l'environnement de la plaque de verre \\
\hline$T_{\mathrm{f}}$ & température fictive \\
\hline$T_{i}$ & température au point de Gauss $i$ \\
\hline$T_{\text {ref }}$ & température de référence \\
\hline$T_{\mathrm{S}}$ & température de la surface de la plaque de verre \\
\hline$T_{\mathrm{V}}$ & température d'un point du volume de la plaque de verre \\
\hline$T_{\mathrm{VFT}}$ & paramètre de la loi de Vogel-Fulcher-Tamman \\
\hline$w_{i j}$ & poids des fonctions de relaxation visqueuse \\
\hline$w_{\mathrm{s} j}$ & poids des fonctions de relaxation structurale \\
\hline$x_{2}$ & coordonnée selon l'épaisseur de la plaque de verre \\
\hline$\Phi$ & facteur de décalage en température \\
\hline$\Phi_{\mathrm{S}}$ & flux radiatif émis par la surface de la plaque de verre \\
\hline$\Phi_{\mathrm{V}}$ & flux radiatif émis par le volume de la plaque de verre \\
\hline$\beta_{\mathrm{g}}$ & coefficient de dilatation du verre « solide $»$ \\
\hline$\beta_{1}$ & coefficient de dilatation du verre « liquide » \\
\hline$\varepsilon_{\mathrm{s}}$ & émissivité de surface \\
\hline$\varepsilon_{\mathrm{v}}$ & émissivité de volume \\
\hline$\eta$ & viscosité à la température $T$ \\
\hline$\eta_{\mathrm{ref}}$ & viscosité à la température $T_{\text {ref }}$ \\
\hline$\rho$ & masse volumique de l'aluminium \\
\hline$\sigma$ & constante de Stéfan-Boltzmann \\
\hline$\sigma_{11}, \sigma_{33}$ & contraintes normales dans le plan de la plaque de verre \\
\hline$\tau_{i j}$ & temps des fonctions de relaxation visqueuse \\
\hline$\tau_{\mathrm{s} j}$ & temps des fonctions de relaxation structurale \\
\hline$\xi$ & temps réduit \\
\hline$\psi_{i}$ & fonctions de relaxation visqueuse \\
\hline
\end{tabular}

\section{Introduction}

L'utilisation du verre structurel dans le bâtiment, c'est-à-dire la réalisation de poutres, poteaux, contreventements en verre à haute résistance nécessite d'une part, le renforcement du verre silico-sodo-calcique par trempe thermique ou chimique et, d'autre part, l'assemblage des plaques de verre par l'intermédiaire de connecteurs métalliques de type boulon, traversant les plaques à joindre, pour l'obtention de poutres longues ou à grande inertie.

Le verre trempé est un matériau précontraint car le traitement thermique de trempe induit des contraintes résiduelles. Ces contraintes n'étant pas mesurables facilement en tout point de l'élément, des simulations de trempe sont indispensables pour connaître les états de contraintes transitoires et résiduelles. Le cas de chargement étudié, une force exercée par le connecteur dans le 


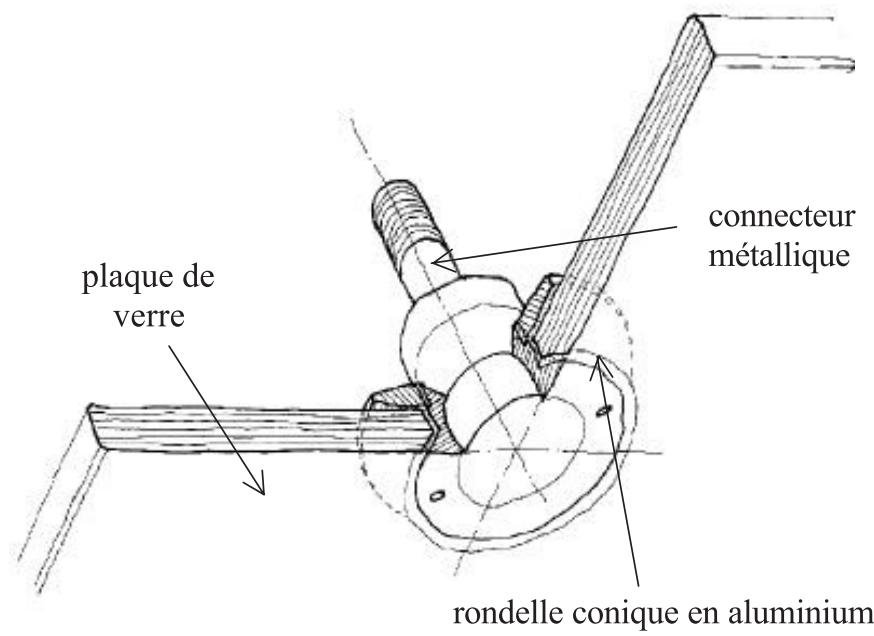

Fig. 1. Schéma de l'assemblage (de type VEA).

plan de la plaque de verre, conduit à une rupture à partir des bords droits ou ceux des trous de liaison. Les simulations par la méthode des éléments finis doivent donc être tridimensionnelles pour prendre en compte l'effet de bord induit par la trempe.

La connaissance des contraintes transitoires est éventuellement intéressante, cet aspect ne sera pas traité dans cet article mais le lecteur pourra se référer à [1] pour la connaissance de l'influence du coefficient de convection forcée et surtout de la température initiale de la plaque de verre sur la contrainte transitoire de tension éventuellement à l'origine de la rupture en cours de trempe d'une plaque de verre.

Les études numériques précédentes de la trempe thermique ont concerné les plaques infinies (calcul 1D), c'està-dire loin des zones bords [2]. Le calcul des contraintes résiduelles de trempe près des bords droits (calcul 2D) a été mené dans [3] et près des trous dans [4] mais les transferts thermiques n'ont pas été traités de façon exhaustive.

Ce papier insiste plus particulièrement sur l'identification des différents échanges thermiques pendant le processus de trempe nécessaire pour la connaissance de l'histoire de la température et du comportement du matériau en tout point de la plaque.

Le type d'assemblage étudié s'inspire de ceux utilisés pour assembler le Verre Extérieur Accroché (VEA) utilisé en façades (Fig. 1). La présente étude concerne la prédiction des contraintes résiduelles près des bords droits mais aussi près de trous chanfreinés dont la géométrie est donnée dans le tableau 1 et la figure 2. Le verre Planilux, produit par Saint-Gobain, a $19 \mathrm{~mm}$ d'épaisseur. Cette étude permet de connaître quelle géométrie permet d'obtenir le meilleur renforcement dans la zone d'assemblage.

\section{Comportement mécanique du verre}

La trempe thermique consiste à refroidir brutalement par jets d'air la plaque chauffée au-delà de $600{ }^{\circ} \mathrm{C}$ (Fig. 3). Ce traitement confère à l'élément un état de contraintes

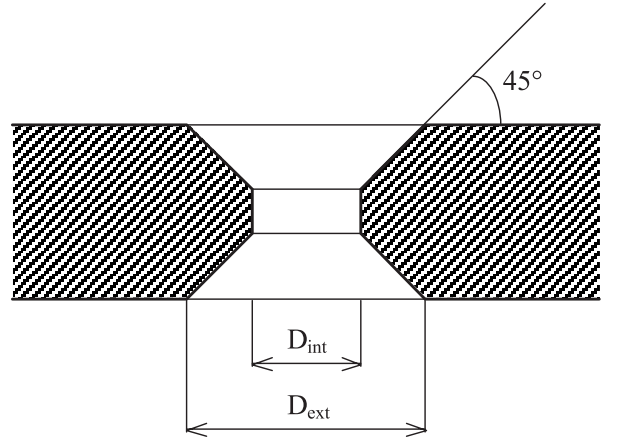

Fig. 2. Coupe d'un trou chanfreiné.

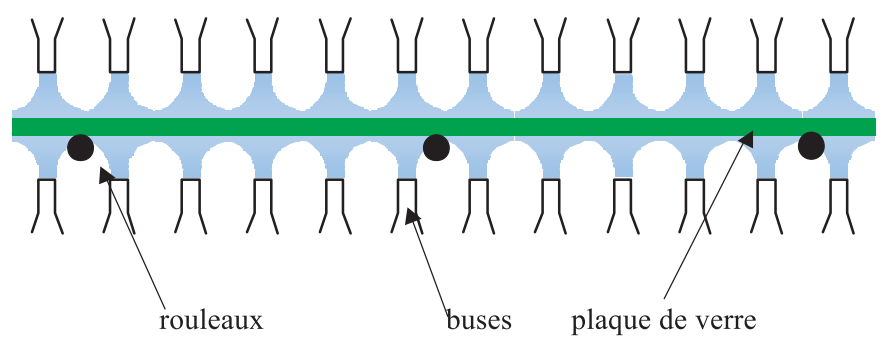

Fig. 3. Trempe thermique horizontale.

Tableau 1. Les cinq géométries de trous étudiées.

\begin{tabular}{ccc}
\hline Désignation & $D_{\text {int }}(\mathrm{mm})$ & $D_{\text {ext }}(\mathrm{mm})$ \\
\hline$a 1$ & 38 & 40 \\
$a 2$ & 54 & 56 \\
$b 1$ & 24 & 40 \\
$b 2$ & 40 & 56 \\
$c 1$ & 30 & 40 \\
\hline
\end{tabular}

résiduelles de traction en cour et de compression en surface. Cela permet d'augmenter la résistance mécanique du verre soumis à de la traction ou à de la flexion. Pendant la trempe, le comportement du verre varie rapidement autour de la température de transition vitreuse $\left(\approx 580{ }^{\circ} \mathrm{C}\right)$ entre les états « solide » et « liquide ». Dans la zone de transition, le verre est un matériau viscoélastique thermo-rhéologiquement simple. Le phénomène de relaxation structurale doit être pris en compte dans la modélisation du procédé de trempe.

Ce comportement mécanique a été largement étudié dans la littérature. Le modèle de Narayanaswamy [5], qui inclut les phénomènes de relaxation d'origines structurale et visqueuse, fait figure de référence; il a été implanté dans le logiciel Abaqus pour cette étude.

\subsection{Comportement viscoélastique du verre}

À température constante, le comportement mécanique du verre est décrit ici en relaxation des contraintes. Le modèle généralisé de Maxwell est un modèle rhéologique discret qui semble bien adapté pour la description du comportement visqueux du verre. Les modules de relaxation de cisaillement $(G)$ et volumique $(K)$ sont écrits avec les 
modules instantanés et différés et développés en séries de Prony à six termes [6]. Le module de cisaillement différé vaut zéro

$$
\begin{gathered}
G(t)=2 G_{\mathrm{g}} \Psi_{1}(t) \\
K(t)=3 K_{\mathrm{e}}-\left(3 K_{\mathrm{e}}-3 K_{\mathrm{g}}\right) \Psi_{2}(t) \\
\Psi_{i}(t)=\sum_{i=1}^{6} w_{i j} \exp \left(-\frac{t}{\tau_{i j}}\right) \quad i=1,2
\end{gathered}
$$

\subsection{Influence de la température}

Quand sa structure est stabilisée, le verre est un matériau thermo-rhéologiquement simple : le matériau présente les mêmes comportements à différentes températures, seule la vitesse des mécanismes microstructuraux de réorganisation varie avec la température. Ainsi la connaissance du comportement mécanique à une température de référence $\left(T_{\text {ref }}\right)$ permet sa connaissance à n'importe quelle température $(T)$. L'influence de la température est introduite au moyen du temps réduit $(\xi)$ défini par l'intermédiaire du facteur de décalage $(\Phi)$ qui est le rapport de la viscosité $(\eta)$ à la température $(T)$ et de la viscosité $\left(\eta_{\text {ref }}\right)$ à la température de référence $\left(T_{\text {ref }}\right)$ [7]

$$
\begin{gathered}
\mathrm{d} \xi=\Phi(T) \mathrm{d} t \\
\Phi(T)=\frac{\eta(T)}{\eta_{\mathrm{ref}}}
\end{gathered}
$$

La dépendance en température de la viscosité $(\eta)$ est supposée suivre une loi VFT [8]

$$
\log \eta=A+\frac{B}{T-T_{\mathrm{VFT}}}
$$

\subsection{Relaxation structurale}

Pendant la trempe, pour des températures proches de la température de transition vitreuse, la structure du verre n'est pas stabilisée. Ainsi, le verre peut avoir plusieurs états pour la même température en fonction de la vitesse de refroidissement, c'est la relaxation structurale.

Ce phénomène est pris en compte par l'intermédiaire du concept de température fictive $\left(T_{\mathrm{f}}\right)$ qui représente la température du verre liquide qui est dans le même état structural que le verre « solide » considéré à la température $(T)$ [9]. Par analogie avec la relaxation visqueuse, une fonction relaxation de volume $\left(M_{\mathrm{v}}(\xi)\right)$ est définie et développée en série de Prony. Sa dépendance avec la température est prise en compte grâce au temps réduit $(\xi(t))$ :

$$
\begin{gathered}
T_{\mathrm{f}}(t)=T(t)-\int_{0}^{t} M_{\mathrm{v}}\left[\xi(t)-\xi\left(t^{\prime}\right)\right] \frac{\mathrm{d} T\left(t^{\prime}\right)}{\mathrm{d} t^{\prime}} \mathrm{d} t^{\prime} \\
M_{\mathrm{v}}(\xi)=\sum_{i=1}^{6} w_{\mathrm{s} i} \exp \left(-\frac{\xi}{\tau_{\mathrm{s} i}}\right)
\end{gathered}
$$

Tous les paramètres dépendant de la température sont fonction de la température fictive. La dilatation thermique est :

$$
\varepsilon_{\mathrm{th}}=\beta_{\mathrm{g}}\left(T(t)-T_{\mathrm{f}}(t)\right)+\beta_{\mathrm{l}}\left(T_{\mathrm{f}}(t)-T_{0}\right)
$$

Le facteur de décalage est :

$$
\begin{aligned}
\ln \Phi\left(T, T_{\mathrm{f}}\right) & =\ln \frac{\eta\left(T, T_{\mathrm{f}}\right)}{\eta_{\mathrm{ref}}} \\
= & B\left(\frac{1}{T\left(1-\frac{T_{\mathrm{VFT}}}{T_{\mathrm{f}}}\right)}-\frac{1}{T_{\mathrm{ref}}-T_{\mathrm{VFT}}}\right) \times \log 10
\end{aligned}
$$

\subsection{Propriétés thermomécaniques du verre silico-sodo-calcique}

Tous les paramètres suivants ont été identifiés par Saint-Gobain Recherche, ils sont donnés dans les tableaux 2 et 3 .

\section{Identification des phénomènes d'échanges thermiques pendant la trempe}

La prise en compte correcte des échanges thermiques est nécessaire pour le calcul des contraintes résiduelles. Ces échanges ont lieu par conduction, convection et rayonnement en raison de la température élevée au début du processus de trempe. Les propriétés thermiques varient avec la température. Cette dépendance, identifiée par Saint-Gobain Recherche, est donnée dans le tableau 4.

Le refroidissement par soufflage de l'air est modélisé par une convection forcée. Loin des bords, la convection forcée est caractérisée par un coefficient unique et par la température de l'air. Pour la modélisation des échanges thermiques autour des zones bord, plusieurs coefficients de transfert convectif sont définis (dans le trou cylindrique, sur la partie chanfreinée, sur les bords droits...).

\subsection{Modélisation du rayonnement}

Le rayonnement est un phénomène complexe dans le verre qui est un milieu semi-transparent. Pendant la trempe, il y a échange de chaleur non seulement entre la surface du verre et les surfaces extérieures, mais aussi entre son volume et l'extérieur, alors que pour la plupart des autres matériaux du génie civil, les ondes sont arrêtées par les premières couches moléculaires qu'elles rencontrent.

Pour un rayonnement provenant d'une plaque infinie, la modélisation retenue consiste à séparer le rayonnement en deux flux issus, d'une part, des surfaces, et d'autre 
Tableau 2. Propriétés thermo-mécaniques du verre.

\begin{tabular}{lll}
\hline Module de Young $E(\mathrm{GPa})$ & $-4,916 \times 10^{6} T+7,1 \times 10^{10}$ & $(T<842,2 \mathrm{~K})$ \\
& $-3,61 \times 10^{7} T+9,67 \times 10^{10}$ & $(T>842,2 \mathrm{~K})$ \\
& 0,22 & \\
Coefficient de Poisson $\nu$ & 0,3 \\
$K_{\mathrm{e}} / K_{\mathrm{g}}$ & $9 \times 10^{-6}$ et $32 \times 10^{-6}$ \\
$\beta_{\mathrm{g}}^{\circ}$ et $\beta_{\mathrm{l}}\left(\mathrm{K}^{-1}\right)$ & $\log (\eta)=-2,74+\frac{4520}{(T-525)}$ \\
$\eta($ loi VFT) (Pa.s) & \\
\hline
\end{tabular}

Tableau 3. Relaxations structurale et visqueuse - poids et temps de relaxation $\left(T_{\text {ref }}=776,51 \mathrm{~K}\right)$.

\begin{tabular}{|c|c|c|c|c|c|c|}
\hline$i$ & 1 & 2 & 3 & 4 & 5 & 6 \\
\hline$\overline{w_{1 i}}$ & 0,0438 & 0,0611 & 0,0899 & 0,2516 & 0,2974 & 0,2561 \\
\hline$\tau_{1 i}(\mathrm{~s})$ & 19 & 291,9 & 1843 & 11800 & 49490 & 171700 \\
\hline$w_{2 i}$ & 0,0307 & 0,0428 & 0,0629 & 0,1761 & 0,2082 & 0,1793 \\
\hline & $w_{\mathrm{s} i}$ & $w_{1 i}$ & $\tau_{2 i}=6 \tau$ & ${ }_{i} ; \quad \tau_{\mathrm{s} i}$ & $=9 \tau_{1 i}$ & \\
\hline
\end{tabular}

part, des points du volume. Ainsi, des émissivités de surface et de volume sont définies :

- l'émissivité de surface $\left(\varepsilon_{\text {surf }}\right)$ correspond au domaine spectral pour lequel le verre est opaque. Soit $\left(T_{\text {ext }}\right)$ la température de l'environnement, le flux radiatif issu de la surface à la température $\left(T_{\mathrm{S}}\right)$ est :

$$
\Phi_{\mathrm{s}}=2 \sigma\left[\varepsilon_{\mathrm{s}}\left(T_{\mathrm{s}}\right) T_{\mathrm{s}}^{4}-\varepsilon_{\mathrm{s}}\left(T_{\text {ext }}\right) T_{\text {ext }}^{4}\right]
$$

- l'émissivité de volume $\left(\varepsilon_{\mathrm{vol}}\right)$ correspond au domaine spectral pour lequel le verre est semi-transparent. Le rayonnement étant issu de tout point du volume, l'émissivité dépend de l'épaisseur (e) de la plaque de verre. Le flux radiatif, supposé uniforme dans l'épaisseur de la plaque de température moyenne $\left(T_{\mathrm{v}}\right)$, est :

$$
\Phi_{\mathrm{v}}=2 \sigma\left[\varepsilon_{\mathrm{v}}\left(e, T_{\mathrm{v}}\right) T_{\mathrm{v}}^{4}-\varepsilon_{\mathrm{v}}\left(e, T_{\text {ext }}\right) T_{\text {ext }}^{4}\right]
$$

Le facteur 2 correspond aux échanges avec les demiespaces au-dessus et au-dessous de la plaque, il est donc supposé que chaque point n'échange de l'énergie par rayonnement qu'avec l'extérieur de la plaque et pas avec les points voisins du volume. Les émissivités de surface et de volume ont été identifiées à partir de mesures expérimentales. Compte tenu des hypothèses simplificatrices, elles doivent être considérées comme des émissivités apparentes du verre plat de $19 \mathrm{~mm}$ [10]. Leur dépendance en température est donnée dans le tableau 5. L'implantation de cette méthode simplifiée dans Abaqus est simple, en chaque point de Gauss $i$, est calculé le flux radiatif émis (respectivement absorbé) en multipliant l'émissivité volumique par la valeur $\left(\sigma T_{i}^{4}\right)$ (respectivement $\sigma T_{\text {ext }}^{4}$ ), et en divisant le produit obtenu par l'épaisseur $(e)$ de la plaque de verre. Pour les mailles de surface, on ajoute le flux correspondant au domaine spectral opaque, qui résulte d'un calcul similaire avec l'émissivité surfacique.

\subsection{Validation du modèle de rayonnement}

Les deux hypothèses de base sont maintenant discutées pour du verre épais de $19 \mathrm{~mm}$ : le rayonnement est supposé uniforme dans l'épaisseur alors qu'il y a en réalité une absorption du rayonnement dépendant de l'épaisseur de la plaque, l'autre question est relative au caractère 1D du modèle de rayonnement valable seulement pour des plaques infinies mais appliqué dans les zones bords.

Ces deux hypothèses ont été validées au moyen d'essais de refroidissement à l'air libre de plaques de verre de $250 \times 250 \times 19 \mathrm{~mm}^{3}$, trouées ou non, chauffées à une température initiale telle que le refroidissement par rayonnement est majeur devant celui par convection libre $\left(T_{0}=640{ }^{\circ} \mathrm{C}\right)$. Durant le refroidissement, les plaques furent filmées avec une caméra Infra-Rouge équipée d'un filtre à $5 \mu \mathrm{m}$ afin de n'enregistrer que le rayonnement émis par la surface car le verre peut être considéré opaque pour cette longueur d'onde, une telle mesure permet donc d'accéder à la température de surface $\left(T_{\mathrm{S}}\right)$ pendant l'essai.

Les contraintes résiduelles dues au refroidissement de la plaque dépendent de l'histoire des températures au sein de celle-ci et donc du rayonnement de volume et de surface. Des informations sur les contraintes résiduelles peuvent être obtenues par mesures photo-élastiques [11]. Le fonctionnement de « l'épibiascope » consiste à exploiter l'effet « mirage » dû à une particularité de fabrication du verre brut de «float» (le verre plat est obtenu par flottaison sur un bain d'étain ce qui a pour conséquence une variation de l'indice de réfraction dans l'épaisseur proche de la face qui a été en contact avec l'étain). L'épibiascope permet ainsi de mesurer les contraintes (ou les déformations car le matériau est élastique) de surface sur la face en contact avec l'étain. Près des bords, une analyse photo-élastique conventionnelle par transmission de lumière monochromatique selon l'épaisseur de la plaque $\left(x_{2}\right)$ avec un « contrôleur de bord » permet de mesure la contrainte de membrane $(\Sigma)$ définie par :

$$
\Sigma=\frac{1}{e} \int_{-e / 2}^{e / 2}\left(\sigma_{33}\left(x_{2}\right)-\sigma_{11}\left(x_{2}\right)\right) \mathrm{d} x_{2}
$$

La contrainte de membrane est nulle dans les zones loin des bords $\left(\sigma_{33}=\sigma_{11}\right)$ mais ne l'est pas près des 
Tableau 4. Conductivité thermique et chaleur spécifique massique du verre.

\begin{tabular}{cc}
\hline Conductivité thermique $\lambda$ & $0,975+8,58 \times 10^{-4}(T-273)$ \\
$(\mathrm{W} / \mathrm{m} . \mathrm{K}, T$ en $\mathrm{K})$ & $1433+6,5 \times 10^{-3} \mathrm{~T}$ \\
\hline Chaleur spécifique massique & \\
du verre liquide $c_{\mathrm{p}, \mathrm{l}}(\mathrm{J} / \mathrm{kg} . \mathrm{K}, T$ en $\mathrm{K})$ & $893+0,4 T-1,8 \times 10^{-7} / T^{2}$ \\
\hline Chaleur spécifique massique & \\
du verre $c_{\mathrm{p}, \mathrm{g}}(\mathrm{J} / \mathrm{kg} . \mathrm{K}, T$ en $\mathrm{K})$ & \\
\hline
\end{tabular}

Tableau 5. Émissivités surfacique et volumique en fonction de la température.

\begin{tabular}{lr}
\hline Émissivité surfacique & $\varepsilon_{\mathrm{s}}=0,8369+1,08807 \times 10^{-4}(T-273)-7,9497 \times 10^{-7}(T-273)^{2}$ \\
$(T$ en $\mathrm{K})$ & $-6,8113 \times 10^{-10}(T-273)^{3}+7,6823 \times 10^{-13}(T-273)^{4}$ \\
\hline Émissivité volumique d'une & $\varepsilon_{\mathrm{v}}=1,0635 \times 10^{-3}-6,5331 \times 10^{-5}(T-273)+1,6486 \times 10^{-6}(T-273)^{2}$ \\
plaque d'épaisseur 19 mm & $-2,3449 \times 10^{-9}(T-273)^{3}+9,3750 \times 10^{-13}(T-273)^{4}$ \\
$(T$ en K $)$ & \\
\hline
\end{tabular}

Tableau 6. Comparaison des contraintes mesurées et calculées sur plaques refroidies en convection libre.

\begin{tabular}{lcc}
\hline Quantités mesurées & Mesure (MPa) & Calcul (MPa) \\
\hline Contrainte de surface & $-67,4$ & $-69,4$ \\
loin des bords et trous & & \\
\hline $\begin{array}{l}\text { Contrainte de membrane } \\
\text { près du bord }\end{array}$ & $-94,4$ & $-91,8$ \\
\hline $\begin{array}{l}\text { Contrainte de surface } \\
\text { près du trou }\end{array}$ & $-73,2$ & $-74,7$ \\
\hline
\end{tabular}

bords en raison notamment de la condition de contrainte normale nulle sur un bord libre.

La simulation numérique des tests de convection libre, avec la prise en compte du rayonnement de surface et de volume, permet de déterminer la température en tout point de la plaque (loin des bords, sur le chanfrein, près du bord droit). Les comparaisons entre les températures calculées et mesurées sont excellentes et sont montrées dans la figure 4 (trou à grand chanfrein).

Les contraintes résiduelles dépendent de l'histoire des températures au sein de la plaque de verre et donc des rayonnements de surface et de volume. L'erreur relative de mesure des contraintes résiduelles par la photo-élasticité est de $10 \%$. La bonne concordance, montrée dans le tableau 6, entre les valeurs de contraintes résiduelles mesurées et celles obtenues par calcul aux éléments finis, permet de valider complètement le modèle d'échanges thermiques par rayonnement.

\subsection{Identification des coefficients de convection forcée}

Les coefficients de convection forcée dans les différentes zones des plaques trouées ont été déterminés à l'aide d'une maquette métallique en aluminium, creuse, représentant la surface extérieure d'une plaque de verre de $40 \times 40 \mathrm{~cm}$ et de $19 \mathrm{~mm}$ d'épaisseur. Chacune des pièces de la maquette a été instrumentée de thermocouples. Les figures 5 et 6 montrent des clichés de cette maquette.

Chaque élément en aluminium (sur le bord, sur la surface de la plaque, différentes faces du trou) est isolé des autres par des rondelles en téflon. Tous les éléments sont instrumentés de thermocouples distribués en différents points de la plaque perforée.

La maquette a ensuite été placée dans des conditions réelles de trempe (sur une ligne de production de la société Saint-Gobain) mais a été portée à une température telle que le rayonnement est négligeable $\left(T_{0}=170{ }^{\circ} \mathrm{C}\right)$. Les thermocouples ont permis de connaître l'historique des températures en différents points de la maquette. Négligeant le terme de conduction dans l'équation de la chaleur (la conductivité de l'aluminium est très élevée), la température est obtenue par l'intermédiaire de la capacité thermique $\left(\rho c_{\mathrm{p}}\right)$, du coefficient de convection forcée $(h)$ et de la température de l'air soufflé $\left(T_{\text {air }}\right)$ :

$$
e \rho c_{\mathrm{p}} \frac{\mathrm{d} T}{\mathrm{~d} t}=h\left(T_{\mathrm{air}}-T\right)
$$

Supposant $(h)$ et $\left(c_{\mathrm{p}}\right)$ constants, l'équation précédente donne l'évolution de la température depuis la température initiale $\left(T_{0}\right)$ :

$$
T=\left(T_{0}-T_{\text {air }}\right) \exp \left(-\frac{h t}{e \rho c_{\mathrm{p}}}\right)+T_{\text {air }}
$$

Les coefficients de convection forcée aux différents points où la température est mesurée sont donc obtenus avec :

$$
h=-\frac{1}{t} e \rho c_{\mathrm{p}} \ln \left(\frac{T-T_{\mathrm{air}}}{T_{0}-T_{\text {air }}}\right)
$$

Tous les coefficients de convection forcée identifiés sont donnés dans le tableau 7 . 

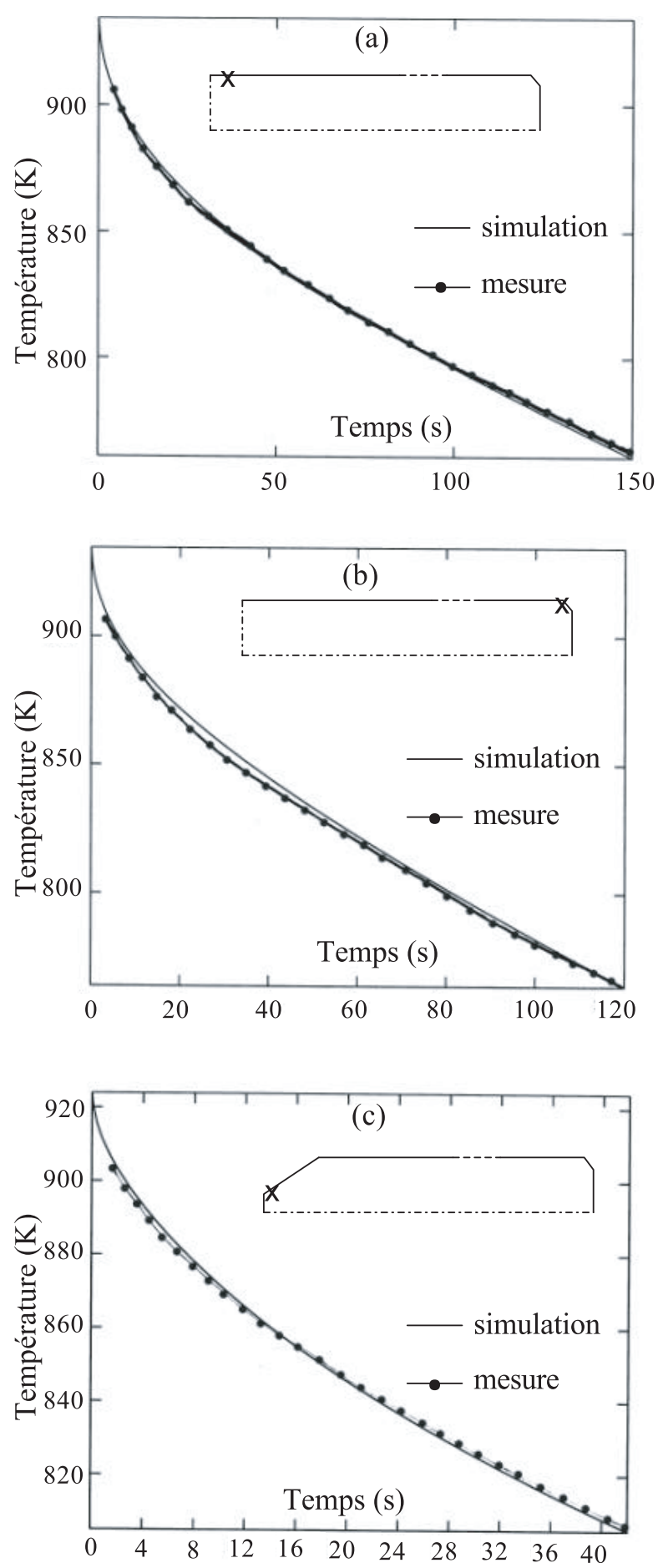

Fig. 4. Comparaisons entre les températures de surface mesurées et calculées : (a) loin des bords, (b) près du bord, (c) sur le chanfrein du trou.

\section{Calcul des contraintes résiduelles}

\subsection{Validation}

Tous les transferts thermiques ont été identifiés. La prédiction des contraintes résiduelles de trempe de plaques de verre épaisses est donc possible.

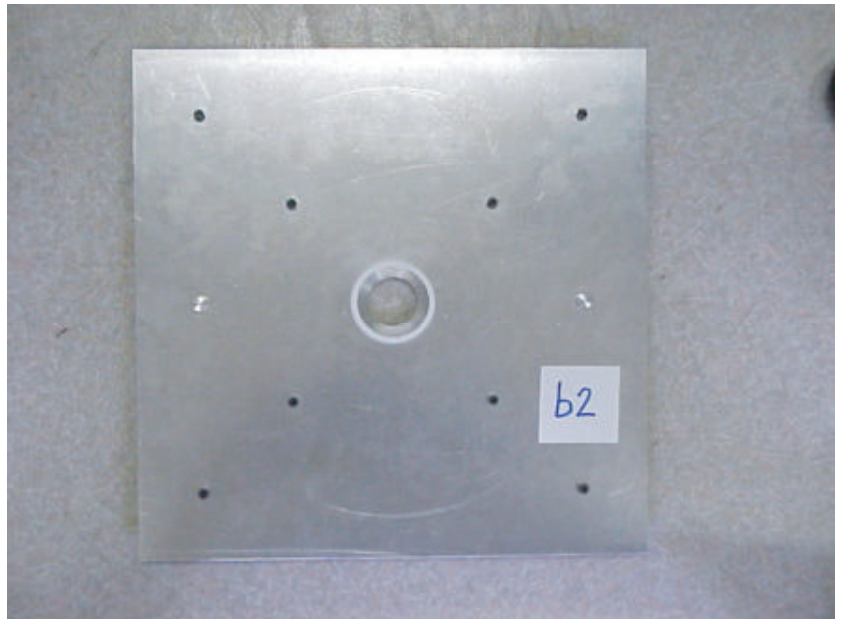

Fig. 5. Maquette creuse en aluminium - trou b2.

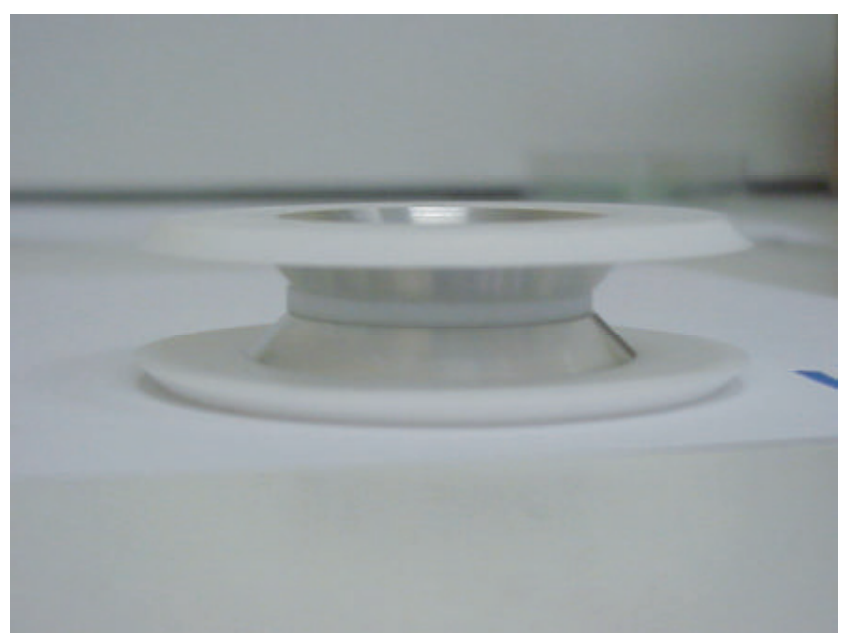

Fig. 6. Zoom sur les constituants du trou chanfreiné - trou b2.

Le rayonnement est pris en compte grâce au modèle à deux émissivités, les coefficients de convection forcée sont ceux identifiés précédemment. La température initiale est fournie par le fabricant $\left(T_{0}=630^{\circ} \mathrm{C}\right)$. La température de l'air a été mesurée pendant les tests précédents $\left(T_{\text {air }}=\right.$ $T_{\text {ext }}=20^{\circ} \mathrm{C}$ ).

50 plaques trouées en verre trempé ont été analysées. Leurs contraintes résiduelles ont été mesurées pour valider la modélisation par éléments finis. Les comparaisons ont porté sur :

- les contraintes de surface loin des zones bords, elles furent mesurées avec l'épibiascope,

- les contraintes de membrane près du trou ou du bord de la plaque, elles furent mesurées par photo-élasticité en utilisant la lumière transmise (le trou a été rempli d'un liquide de même indice de réfraction que le verre).

Une estimation de ces contraintes avec moins de $10 \%$ d'erreur a été obtenue. Le tableau 8 , les figures 7 et 8 montrent le bon accord entre les contraintes prédites et mesurées. Ces résultats permettent de valider le modèle développé pour la prédiction des contraintes résiduelles au 
Tableau 7. Différentes valeurs identifiées des coefficients de convection forcée.

\begin{tabular}{|c|c|c|c|c|c|c|c|}
\hline \multirow{2}{*}{\multicolumn{2}{|c|}{$\begin{array}{l}\text { Faces de la plaque } \\
\qquad\left(\mathrm{W} / \mathrm{m}^{2} . \mathrm{K}\right)\end{array}$}} & \multicolumn{5}{|c|}{ Faces du trou $\left(\mathrm{W} / \mathrm{m}^{2} . \mathrm{K}\right)$} & \multirow{3}{*}{$\begin{array}{c}\text { Bords droits } \\
\left(\mathrm{W} / \mathrm{m}^{2} \cdot \mathrm{K}\right)\end{array}$} \\
\hline & & \multicolumn{3}{|c|}{ chanfrein } & \multicolumn{2}{|c|}{ cylindre } & \\
\hline Loin du trou & Près du trou & $b 1$ & $b 2$ & $c 1$ & $a 1$ & $a 2$ & \\
\hline 77 & 72 & 74 & 78 & 75 & 58 & 61 & 62 \\
\hline
\end{tabular}

Tableau 8. Comparaisons entre les contraintes de surface prédite et mesurée loin des bords.

\begin{tabular}{cc}
\hline Mesurée avec l'épibiascope & Calcul \\
\hline$-147 \mathrm{MPa}$ & $-144 \mathrm{MPa}$ \\
\hline
\end{tabular}

sein de plaques de verre trouées et trempées. Le modèle peut maintenant être utilisé pour l'optimisation des assemblages en recherchant quelle géométrie permet d'avoir le meilleur renforcement par trempe thermique.

\section{Application}

Les éléments de comparaisons suivants ont été considérés pour l'analyse du renforcement du verre près du trou :

- les contraintes de surface circonférentielles dans le trou,

- les contraintes de membrane au voisinage du trou,

- la ligne neutre : au-delà de cette ligne, une zone de contrainte de membrane de tension apparaît, cela peut fragiliser le trou,

- l'épaisseur de la « peau » en compression au voisinage du trou.

Les comparaisons des différentes géométries de trou étudiées sont montrées dans les figures 9 et 10 et dans le tableau 9. Ces résultats permettent de conclure que le meilleur renforcement est obtenu pour les trous à larges chanfreins.

\section{Conclusion}

La prédiction de la résistance d'assemblage dans les structures en verre trempé thermiquement nécessite une modélisation par éléments finis du processus de trempe pour un calcul des contraintes résiduelles au voisinage des assemblages. Pour cette analyse, il est apparu nécessaire de modéliser correctement les transferts thermiques pendant la trempe, notamment la convection forcée et le rayonnement. Les transferts radiatifs ont été pris en compte par l'intermédiaire d'un modèle à deux émissivités. La pertinence de cette modélisation du rayonnement pour le verre structural a été vérifiée expérimentalement. Les coefficients de convection forcée

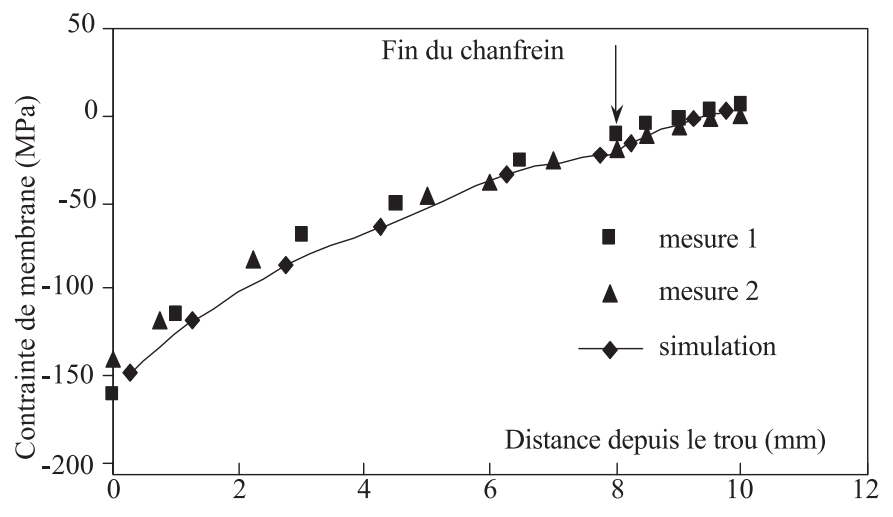

Fig. 7. Comparaisons entre les contraintes de membrane calculées et mesurées près d'un trou à large chanfrein.

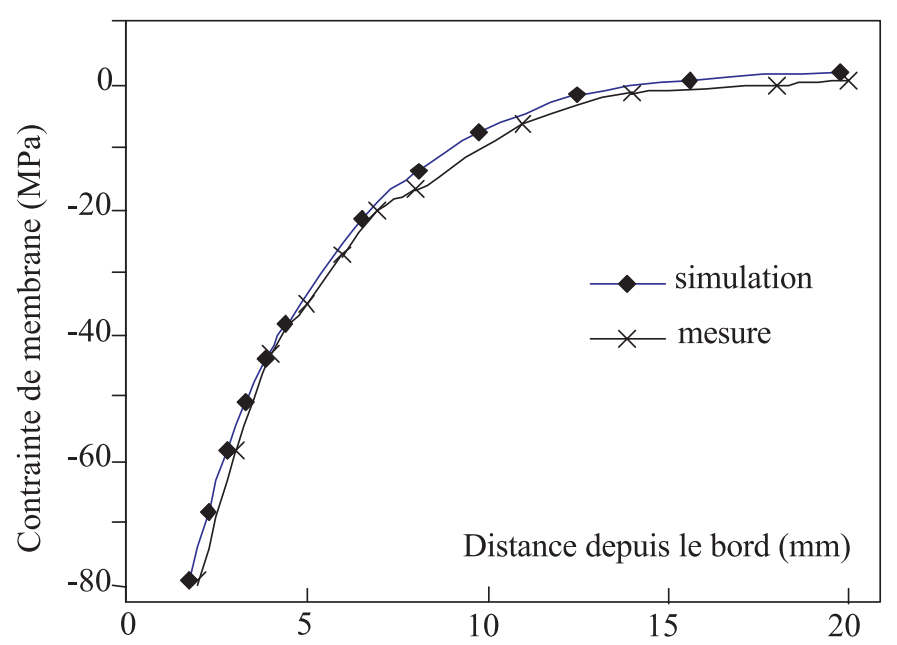

Fig. 8. Comparaisons entre les contraintes de membrane calculées et mesurées près d'un bord droit (les mesures ont débuté près du chanfrein).

ont été identifiés par l'intermédiaire de maquettes en aluminium soumises à des conditions réelles de trempe. Ces coefficients sont différents loin des zones bords et au voisinage des trous ou bords droits. L'identification de ces transferts de chaleur, combinée à l'utilisation du modèle de Narayanaswamy, permet de conclure que le processus de trempe thermique est le plus efficace pour les trous avec larges chanfreins. 

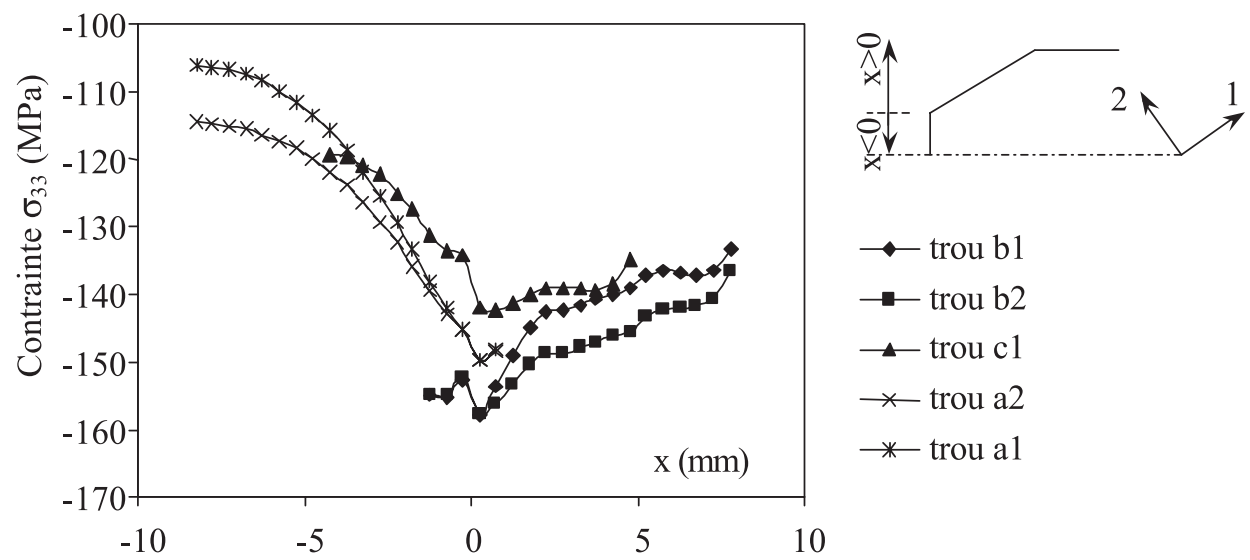

$\longrightarrow$ trou b1

$\because$ trou b2

$\rightarrow$ trou $\mathrm{c} 1$

$\times$ trou a2

$\rightarrow$ trou al

Fig. 9. Contrainte de surface $\sigma_{33}$ pour chacune des géométries de trou - la plus grande compression est obtenue pour b2.

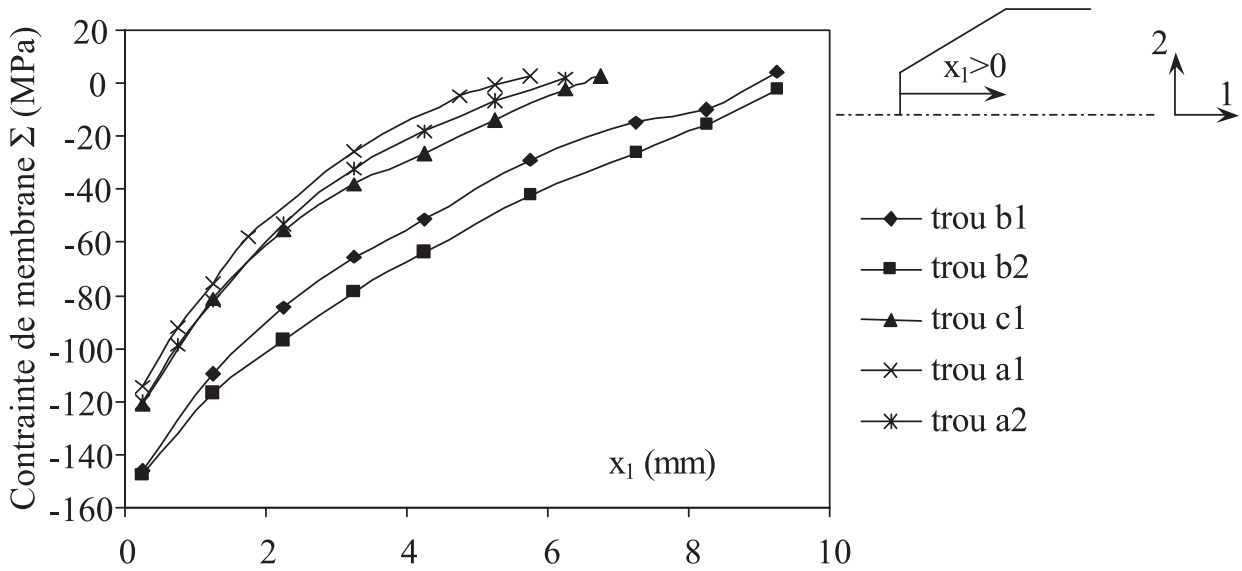

Fig. 10. Contrainte de membrane $\Sigma=\frac{1}{e} \int_{-e / 2}^{e / 2}\left(\sigma_{33}\left(x_{2}\right)-\sigma_{11}\left(x_{2}\right)\right)$ d $x_{2}$ pour chacune des géométries de trou - la plus grande compression est obtenue pour b2.

Tableau 9. Récapitulatif des prédictions de contraintes résiduelles sur chacun des trous - le trou b2 permet d'obtenir le meilleur renforcement.

\begin{tabular}{|c|c|c|c|c|c|c|}
\hline \multirow{3}{*}{ Trou } & \multicolumn{3}{|c|}{ Contrainte de surface $\sigma_{33}$} & \multirow{3}{*}{$\begin{array}{l}\text { Contrainte } \\
\text { de membrane } \\
\left(x_{1}=0\right)(\mathrm{MPa})\end{array}$} & \multirow{3}{*}{$\begin{array}{l}\text { Position de la } \\
\text { ligne neutre } \\
\text { (mm) }\end{array}$} & \multirow{3}{*}{$\begin{array}{c}\text { Épaisseur en } \\
\text { compression (mm) }\end{array}$} \\
\hline & \multicolumn{3}{|c|}{$(\mathrm{MPa})$} & & & \\
\hline & Min. & Moy. & Max. & & & \\
\hline$a 1$ & $-106,1$ & -123 & $-149,8$ & $-119,6$ & 5,38 & 3,00 \\
\hline$a 2$ & $-114,6$ & $-127,7$ & $-149,7$ & $-125,1$ & 6,08 & 3,35 \\
\hline$b 1$ & $-133,1$ & $-143,8$ & $-157,6$ & $-155,2$ & 8,94 & 3,70 \\
\hline$b 2$ & $-136,6$ & $-147,9$ & $-157,7$ & $-155,6$ & 9,49 & 4,00 \\
\hline$c 1$ & $-119,2$ & $-133,1$ & $-142,4$ & $-131,4$ & 6,50 & 3,64 \\
\hline
\end{tabular}




\section{Références}

[1] H. Carré, L. Daudeville, Analysis of residual stresses and of the load-bearing capacity of tempered glass plates, in : S. Denis (éd), Proceedings of European Conference on Residual Stresses ECRS4, ENSAM Cluny, France, 1996

[2] O.S. Narayanaswamy, R. Gardon, Calculation of residual stresses in glass, J. Am. Ceramic Soc. 52(10) (1969) $554-558$

[3] H. Carré, L. Daudeville, Load bearing capacity of tempered structural glass, ASCE J. Eng. Mech. 125(8) (1999) 914-921

[4] W. Laufs, G. Sedlacek, Stress distribution in thermally tempered glass panes near the edges, corners and holes, Part 1, Temperature distributions during the tempering process of glass panes, Glass Science and Technology 72(1) (1999) 1-14

[5] O.S. Narayanaswamy, A model of structural relaxation in glass, J. Am. Ceramic Society 54(10) (1971) 491-498
[6] R. Gy, L. Duffrene, M. Labrot, New insights into the viscoelasticity of glass, J. Non-Cryst. Solids 175 (1994) 103-117

[7] F. Schwarzl, A.J. Staverman, Time-temperature dependance of linear viscoelastic behavior, J. Appl. Phys. 23(8) (1952) 838-843

[8] L. Duffrene, Comportement viscoélastique d'un verre silico-sodocalcique dans le domaine des températures intermediaires, Thèse de l'École nationale supérieure des mines de Paris, Paris, France, 1994

[9] A.Q. Tool, Relation between inelastic deformability and thermal expansion of glass in its annealing range, J. Am. Ceramic Soc. 29(9) (1946) 240-253

[10] D. Banner, S. Klarsfeld, Influence of composition upon the apparent conductivity and emissivity of glass as a function of thickness and temperature, Thermal conductivity 21, Cremers and Fine, Plenum Press, New York, USA, 1990

[11] H. Aben, C. Guillemet, Photoelasticity of glass, SpringerVerlag, Berlin, 1993 